\title{
Forward and backward masking as a function of relative overlap and intensity of test and masking stimuli'
}

PETER H. SCHILLER

MASSACHUSETTS INSTITUTE OF TECHNOLOGY

Foru'ard and backward vicual masking for patterns was investigated as a function of relative overlap between test and masking stimuli, relative intensity of masking stimulus and length of interval between stimuli. The extent of masking increased with increasing spatial overlap between stimuli and with increasing intensity of the masking stimulus. Increasing the interval between the stimuli decreased masking; this occurred at faster rates in backward than in forward masking. Possible mechanisms explaining these findings are discussed.

The investigation of visual masking has currently been expanded to explore the interaction between relatively complex patterns, such as letters and words (Kinsbourne \& Warrington, 1962; Schiller, 1965a; Schiller \& Wiener, 1963; Sekuler, 1965; Sperling, 1963a). This kind of interference, which is observed when two patterns are exposed in rapid succession, has been studied most extensively in backward masking, where the stimulus to be discriminated is shown first and the masking stimulus is shown second. Currently, considerable interest has arisen in forward masking as well, and the question has been raised whether these two forms of masking can be assumed to reflect a single underlying process. Findings favoring such an interpretation were reported by Eriksen and Lappin (1964) who employed a homogeneous masking field in their study. On the other hand, we have found that when the masking stimulus as well as the test stimulus is a pattern, there is a pronounced difference in forward and backward masking (Schiller \& Smith, 1965; Smith \& Schiller, 1966).

In order to determine to what extent patterning influences forward and backward masking, in this study the degree of overlap between test and masking stimuli was systematically varied. To further ascertain the parameters giving rise to forward and backward masking, the effect of stimulus intensity was also investigated.

\section{METHOD}

\section{Subjects}

The Ss were two paid volunteers, SG and $\mathbf{R M}$, with equal visual acuity for both eyes.

\section{Apparafus}

The apparatus was a three-field Dodge-type tachistoscopic viewing box. The electronic programmer driv- ing this viewer was built by Sky Instrument Company. This programmer has a rated accuracy of $\pm 2 \%$. The light sources were cold cathode mercury argon lamps coated with magnesium tungstate phosphor. A "keepalive" electrode in the lamps assured reliable firing characteristics (Kolers, 1962).

\section{Materials}

The stimuli were 18 letters of the alphabet (see Table 1). These letters were contact negatives of No. 115 Futura Medium "'Lettraset" letters. Two sets of negatives were prepared. Each set was mounted on an anodized aluminum disk measuring $10 \mathrm{in.}$ in diameter. Each disk had 20 1-in. holes drilled near its outer edge. The negatives were mounted over these holes. The disks were then mounted in the tachistoscope, one in each of two fields, allowing $\mathrm{E}$ to place the letters in the correct viewing position by turning the disk. Around the outer circumference the disks were notched. An anchored spring-loaded roller made it possible to "click-in" the stimuli to insure accuracy of position. The light sources transilluminating the stimuli were mounted in an enclosure behind the disks. A $1 / 8 \mathrm{in}$. thick white plexiglas cover served as a diffusing surface for the light sources. The third field in the tachistoscope was used as the focusing field in which two small red dots appeared. The letters were presented between these dots. The visual angle of the letters was $.8^{\circ}$ and that of the focusing light $1.5^{\circ}$.

Prior to the experiment letter pairs were selected with different degrees of overlap. This was done by placing each of the letters over a grid and determining the area of the letter and its overlap with other letters. The letters were then paired making up various percentages of overlap as shown in Table 1. This table

Table 1. Test and Masking Stimulus Pairs

Per Cent Overlap of Test Stimuli With Masking Stimuli

\begin{tabular}{ccccccccccccc}
\hline \multicolumn{1}{c}{100} & \multicolumn{2}{c}{$92-85$} & \multicolumn{2}{c}{$75-67$} & \multicolumn{2}{c}{$60-50$} & & \multicolumn{2}{c}{$44-35$} & & 27.14 \\
\hline MS & TS & MS & TS & MS & TS & MS & TS & MS & TS & MS & TS \\
\hline G & C & D & G & V & Y & R & A & U & V & L & Y \\
D & L & B & E & Y & T & Z & K & P & D & A & Z \\
E & F & E & P & S & F & K & A & T & R & V & O \\
O & C & B & S & K & R & V & G & C & B & T & U \\
R & P & D & U & Z & T & S & Z & F & K & A & D \\
E & L & P & F & G & S & C & D & Y & B & L & V \\
\hline
\end{tabular}

$M S=$ Masking Stimulus 
shows the extent to which the test letters were covered by the masking letters. Six categories were obtained, each with different degrees of overlap. The first column of letters for each of the six groups in Table 1 shows the letters which were used as masking stimuli; the second column shows the test stimuli. In forward masking a masking letter always appeared first followed by a test letter; in backward masking a test letter appeared first and was followed by a masking letter. The stimuli were arranged in such a fashion that each letter in the set, with the exception of letters $E$ and $F$, appeared twice as a masking stimulus and twice as a test stimulus. Letter $E$ appeared three times as a masking stimulus and once as a test stimulus; F appeared once as a masking stimulus and three times as a test stimulus. This balancing of letters was carried out in order to forestall the development of possible response biases in the course of the experiment.

\section{Procedure}

Each session throughout the study began with a period of dark-adaptation lasting until $S$ could report the presence of the two focusing lights. This usually took 5-10 min. For the presentation of the stimuli $\mathrm{S}$ was instructed to focus midway between the two red dots.

Prior to the experiment several days were devoted to threshold measurements of letters and viewing of masking phenomena. After S's threshold had become stable the experiment was begun. Threshold measurements were obtained by a method of constant stimuli. The duration of the test and masking letters was set constant at the point at which each $S$ was first able to correctly identify single letters at .19 ft. L $100 \%$ of the time during the last two days of pre-testing. The duration of the test and masking letters for SG was $6 \mathrm{msec}$. each and for RM $7 \mathrm{msec}$. each. This duration was maintained throughout the experiment; measurements taken at the beginning of each day using the method of limits for single letters have shown these thresholds stable.

The test letters during the experiment always appeared at the same level of illumination, which was $.19 \mathrm{ft}$. L. The masking letters were presented at three levels of illumination, $.19,1.9$, and $19 \mathrm{ft}$. L. At each of the luminance levels, for both forward and backward masking, four interstimulus intervals were employed: $5,40,100$, and $200 \mathrm{msec}$., making for a total of 144 presentation conditions ( 2 orders of presentation, 3 luminance levels, 4 interstimulus intervals and 6 degrees of overlap).

The stimuli were presented in pairs $90 \%$ of the time. The remaining $10 \%$ of the time single letters were presented. The S's task throughout the experiment was to identify the letters shown. The $S$ was instructed that at times he would see two letters and at other times only one and that his task was to indicate what letter or letters were shown.
Over a period of 18 sessions 18 measurements were obtained for each condition of presentation making for a total of 2592 measurements per S. During every session one presentation was made for each condition as follows: Half of each session was devoted to forward masking and half to backward masking; the order was reversed from day to day. Intensity was randomized in blocks of 18 , the interstimulus interval was randomized in blocks of 6 and stimulus overlap was randomized by trial.

The interval between presentations was approximately $6 \mathrm{sec}$. The E alerted S by saying "ready" prior to each presentation. A brief rest period was provided between forward and backward masking conditions.

\section{RESULTS}

Most of the data presented here are analyzed in terms of per cent correct identification of test stimuli. The per cent of time two stimuli were reported as seen (correctly or incorrectly) was also analyzed; these data are not presented as they yielded results practically identical to those obtained by analyzing correct responses.

Forward and Backward Masking. The results show that for forward and backward masking the per cent correct identifications were $30.4 \%$ and $53.7 \%$ for observer RM and $34.5 \%$ and $\mathbf{5 7 . 2 \%}$ for observer SG, respectively. This pronounced difference between forward and backward masking can also be seen in Figs. 1 and 2. These data show that at short interstimulus intervals the

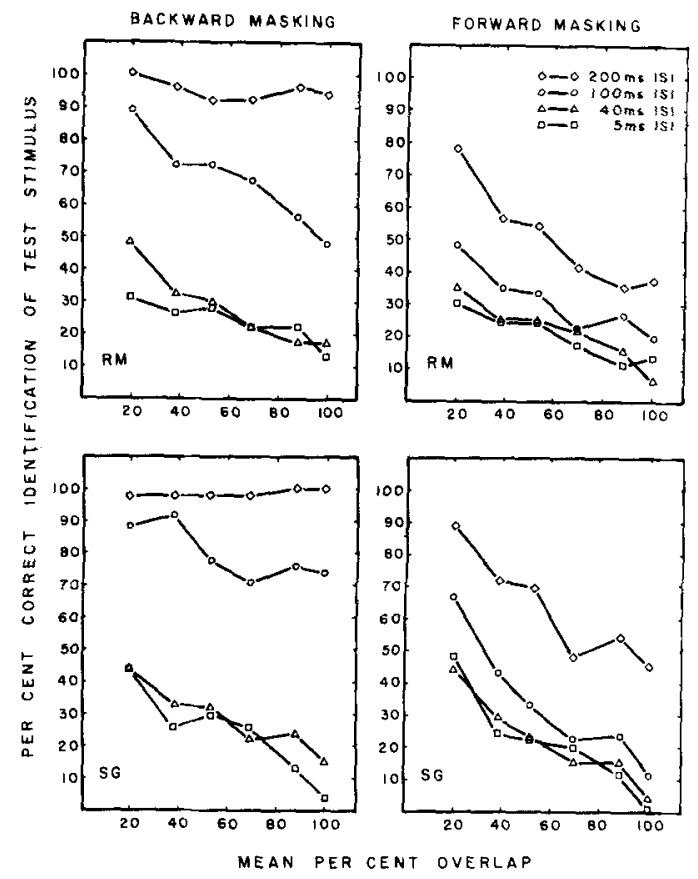

Fig. 1. Per cent correct test stimulus identification in forward and backward masking as a function of overlap and interstimulus interval. 


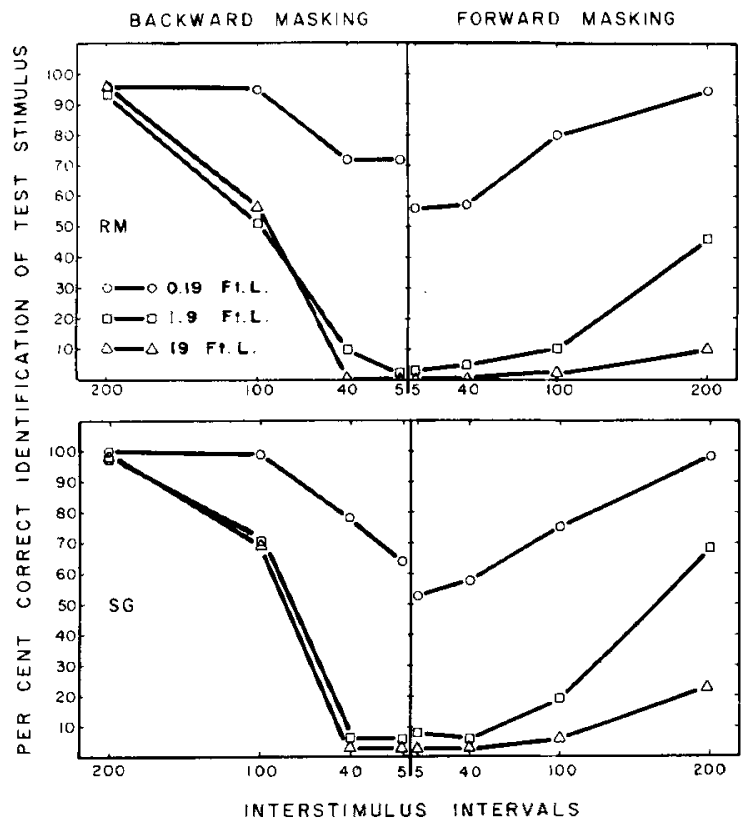

Fig. 2. Per cent correct test stimulus identification in forward and backward masking as a function of masking stimulus intensity and interstimulus interval.

extent of masking is relatively similar in forward and backward masking. The rate at which the extent of masking decreases, however, as the interstimulus interval is increased is slower in forward than in backward masking; at an interstimulus interval of $200 \mathrm{msec}$. there is practically no interference in backward masking, while in forward masking at this level the interference is still considerable.

Overlap Conditions. The per cent correct test stimulus identification for each of the six conditions of overlap in forward and backward masking at each of the four interstimulus interval levels is shown in Fig. 1. For these data the three intensity conditions were combined. The results show that the extent of interference in both forward and backward masking declines monotonically as the per cent overlap is decreased. The amount of interference is approximately the same at an interstimulus interval of 5 and $40 \mathrm{msec}$. and then becomes considerably less at longer interstimulus intervals. The differences in forward and backward masking are pronounced even with the lowest overlap condition.

Intensity. The per cent correct identification of the test stimuli in forward and backward masking for the three intensity conditions is shown in Fig. 2. For this graph the overlap conditions were combined. The data show that increasing the luminance of the masking stimulus decreases the per cent correct identification of the test letters. However, the extent to which intensity influences interference in forward and backward masking is different. In backward masking in- creasing intensity by one log unit has a pronounced effect; yet, increasing it by $2 \mathrm{log}$ units does not seem to produce further interference. In forward masking the overall effects are greater; furthermore, the extent of interference keeps increasing as the intensity of the masking stimulus is increased. These findings, as well as all previous ones, show a high degree of correspondence for the two Ss.

Comparison of Test and Masking Stimulus Identification. The masking stimuli were correctly identified $100 \%$ of the time in both forward and backward masking when these stimuli were presented at 1.9 and $19 \mathrm{ft}$. $\mathrm{L}$.

The per cent correct identification of the test and masking letters when each was presented at .19 ft. $L$. is shown in Fig. 3 for each of the overlap conditions. In this graph the interstimulus intervals are combined. The per cent correct identification of the test stimuli increases monotonically as degree of overlap is decreased. For the masking stimuli a different function is obtained. Per cent identification is lowest at intermediate degrees of overlap. The accuracy in masking stimulus identification at high degrees of overlap seems to be due to the fact that the contours of the two stimuli are quite similar; since the masking stimulus at $100 \%$ overlap is the more extensive figure, it is the one perceived. Thus, at short interstimulus intervals, for example, when $L$ is the test figure and $\mathrm{E}$ is the masking figure, Ss report nearly $100 \%$ of the time seeing only one letter, the letter $\mathrm{E}$. The case is similar for the other stimuli having high degrees of overlap. A mutual interference between test and masking stimuli is not found until there is an intermediate degree of overlap between them. At low degrees of overlap the letters are relatively separate, yielding a high degree of correct identification for both stimuli.

In order to further assess the extent of mutual interference between the two stimuli, the per cent of time both were incorrectly identified was determined. These data show that with both stimuli at .19 $\mathrm{ft}$. L., the per cent incorrect identification for both stimuli was

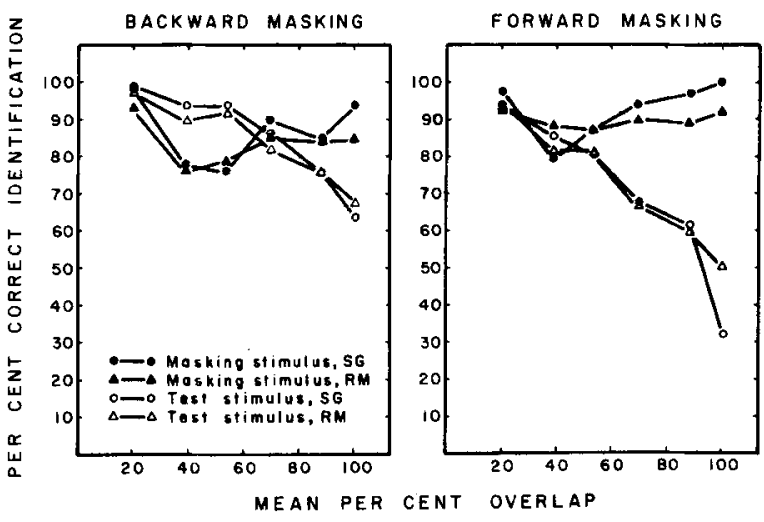

Fig. 3. Per cent correct identification of test and masking stimuli when at equal luminance $(.19 \mathrm{ft}$. $\mathrm{L}$.) as a function of overlap 
only $2.0 \%$ for observer SG and $\mathbf{1 . 5 \%}$ for observer RM. Thus, most of the time at least one letter was correctly identified.

The differences in forward and backward masking are pronounced when (a) the masking stimulus is more intense and (b) when there is an asymmetry of stimulus overlap between test and masking stimuli. In order to test whether or not there is a preferential recognition for the first and second stimulus when other factors are minimized, the test and masking stimulus data were pooled for forward and backward masking at the $.19 \mathrm{ft}$. L. intensity level, and the per cent correct identification for the stimulus appearing first and second was determined. In order to minimize the effects of asymmetries of overlap, the three highest overlap conditions were omitted from this analysis. The results show that the per cent correct recognition for the test stimulus appearing first and second was $90.6 \%$ and $82.8 \%$ for observer RM and $91.6 \%$ and $85.2 \%$ for observer SG respectively. Thus, the frequency of correct recognition is somewhat higher for the first of two successive stimuli. The differences, however, a re sma11, suggesting that the effects observed in forward and backward masking are primarily a function of such variables as relative intensity and overlap.

\section{DISCUSSION}

The results of this study show pronounced differences in forward and backward masking. Not only is masking more effective and more prolonged under conditions of forward masking, but increasing the intensity of the stimulus produces different effects for these two conditions. In backward masking, interference increases only up to a point as intensity of the masking stimulus is increased, while in forward masking, interference continues to increase with intensity. These findings suggest that, with patterns as masking stimuli, interference in forward and backward masking does not reflect the same processes. In forward masking, at least an added component or components must be involved. The after-image produced by the first stimulus may be responsible for some of the interference observed in forward masking.

Several hypotheses have been produced to explain the interference observed in visual masking (Kolers, 1962; Sperling, 1963b). One of these views (Boynton, 1961) holds that information in the nervous system is processed in time quanta. When two stimuli are presented within one quantum they cannotbe processed separately. One of the problems with this view is that the time required for the separate analysis of two stimuli seems to be a function of a number of variables, three of which appear in this study: stimulus order, stimulus intensity and stimulus overlap. The results showed that the time for stimulus processing changed as a function of these variables. In view of the fact that the time quantum appears to be vastly different for different stimulus conditions, the concept of quantum would seem to lose much of its explanatory power.

The data on stimulus overlap show maximal masking with greatest overlap. This implies that masking should be most pronounced when the two stimuli are identical. With the paradigm employed in this study this cannot be tested adequately, since when the same stimulus is shown twice in succession, the task becomes, implicitly at least to detect the break between the two presentations.

Degree of overlap reflects only one aspect in the analysis of masking when patterned stimuli are employed. Other dimensions have been noted, such as the extent of patterning (e.g., number of intersecting contours; Schiller \& Wiener, 1963). The present findings suggest that masking may be maximal when the test and masking stimuli are similar in degree of patterning. However, it may turn out to be difficult to devise experiments which could clearly separate the role of overlap between masking and test stimuli from that of patterning (number of contours).

\section{References}

Alpern, M. Metacontrast. J. Opt. Soc. Amer., 1953, 43, 648-657. Boynton, R. M. Some temporal factors in vision. In W. A. Rosenblith (Ed.), Sensory communication. New York: Wiley, 1961. Pp. $739-756$.

Eriksen, C. W., \& Lappin, J. S. Luminance summation-contrast reduction as a basis for certain forward and backward masking effects. Psychon. Sci., 1964, 1, 313-314.

Fehrer, E., \& Raab, D. Reaction time to stimuli masked by metacontrast. J. exp. Psychol., 1962, 63, 143-147.

Kinsboume, M., \& Warrington, E. K. Further studies on the masking of brief visual stimuli by a random pattern. Quart. J.exp. Psychol., 1962, 14, 235-245.

Kolers, P. A. Intensity and contour effects in visual masking. Tis. Res., 1962, 2, 277-294.

Schiller, P. H. Metacontrast interference as determined by a method of comparisons. Percept. mot. Skills, 1965, 20, 279-285.

Schiller, P. H. Monoptic and dichoptic visual masking by patterns and flashes. J. exp. Psychol., 1965, 69, 193-199.

Schiller, P. H., \& Smith, M. C. Detection in metacontrast. $J$. exp. Psychol., in press.

Schiller, P. H., \& Wiener, M. Monoptic and dichoptic visual masking. J. exp. Psychol., 1963, 66, 386-393.

Sekuler, R. W. Spatial and temporal determinants of risual backward masking. $J$. exp. Psychol., in press.

Sperling, G. A model for visual memory tasks. Human factors, $1963,5,19-31$.

Sperling, G. What visual masking can tell us about temporal factors in perception. XVII. Intcrnational Congress on Prucholory. Proceedings. Washington, D. C., 1963, Amsterdam: North Holland Publishing Company, 1964.

\section{Note}

1. This research was supported, in part, by a Mental Health Postdoctoral Fellowship FPD-11, 589. The author is indebted to Barbara $D$. von Randow for her assistance.

(Received in the Editorial Offic' April 9, 1966,) 